\title{
Knowledge in Digital Decision Support System
}

\author{
Erika Matsak ${ }^{1}$ and Peeter Lorents ${ }^{2}$ \\ ${ }^{1}$ Tallinn University, Narva road 25, 10120, Tallinn, Estonia \\ ${ }^{2}$ Cooperative Cyber Defence Centre of Excellence, Estonia \\ erika.matsak@tlu.ee, peeter.lorents@mil.ee
}

\begin{abstract}
Digital circuits have been studied, which can support the decisionmaking process. The circuits allow realizing two types of inference rules: modus ponens and modus tollens. Inference process uses knowledge that comes from a description of situations, and axioms, which comes from the experts.
\end{abstract}

Keywords: Decision making. Logical deduction. Digital circuits.

\section{Introduction}

Managing real situations requires operating with growing amounts of information during ever decreasing amounts of time. At the same time, the quality of decisions must not suffer. The quality is ensured by using correct situation descriptions and a correct inference process. The latter, in turn, requires the use of correct inference steps, which give a correct result when used with correct premises. This brings us to the problem: how to support the human decision process with suitable technological solutions that would enable operation with large information amounts during a short amount of time. These suitable technological solutions can nowadays be divided into two broad categories: software and hardware solutions. The credibility of these solutions is based on corresponding theoretical work.

For example, when discussing decision processes, we can refer to situation description [1], event description [2], decision trees [3], statistical methods [4], etc.

In several cases, (custom built) hardware solutions have proven to be faster and more reliable than corresponding software solutions. For example, this has been claimed about neural networks. Based on this, it is interesting to study the use of digital circuits for implementing the correct logic inference steps needed in the decision process. This is the area that we cover in this publication.

\section{Description, Formulas, Knowledge - General Overview}

A substantial part of situation descriptions is formed by statements that generally contain the following content:

- Some object has or lacks a certain property.

- Some objects have or lack a certain relation. 
In logic, such statements can be represented by so called atomic formulas or their negations. These formulas can be (in the frame of classical logic) either true or false, depending on whether the describer has correctly understood the state of affairs.

Often the situation descriptions do not contain everything that we need to know or should know.

Example. While describing a disaster area or combat zone, one observer has determined that it is possible to get from location $\mathrm{X}$ to location $\mathrm{Y}$. Another observer has determined that it is possible to get from location $\mathrm{Y}$ to location $\mathrm{Z}$. If the decision maker

- knows that the binary relation R, which represents the existence of a safe route between two locations, is transitive (meaning $\mathrm{R}(\mathrm{X}, \mathrm{Y}) \& \mathrm{R}(\mathrm{Y}, \mathrm{Z}) \supset$ $\mathrm{R}(\mathrm{X}, \mathrm{Z}))$, and

- can apply the inference step that comes from a rule called modus ponens $\frac{\mathrm{P} \quad \mathrm{P} \supset \mathrm{Q},}{\mathrm{Q}}$

then he can state that it is possible to get from location $\mathrm{X}$ to location $\mathrm{Z}$ and he can make the corresponding decision.

We can guess that until that application of the inference step, the decision maker did not know that it is possible to get from $\mathrm{X}$ to $\mathrm{Z}$. But now he knows. More than that, he knows something that actually meets the real conditions in the context of the situation at hand, without having to observe it locally and without having information about this specific case. It is a good demonstration of the need for logically correct inferences: it often allows reliably acquiring missing parts of the situation description, even if there is no direct source for the correct information.

When relating knowledge with formulas [5], we should presume that if in some situation some aspects are not as they were reported, then the truth value of the formula with the corresponding knowledge should be 0 . However, if everything "checks out", then the truth value should be, for example, 1. (We are using expressions like "should be 0 " and "for example, 1", since the truth values do not have to be numbers! For example, we can also use the capital first letters of the words false and true, or even open subsets of some topological space [6]).

In real situations, it is often possible to build a formula based on various pieces of available information, but we cannot verify its correctness. In other words, we may have the formula, but it lacks (for the moment) a truth value of true or false. In such situations it may be useful to use so called tri-valent logic [see, for example, 7, 8, 9], where a third truth value is used (for example, not determined or indeterminable).

Therefore, we can say that the decision maker may possess knowledge (for example, from observer reports) and corresponding formulas, of which

- some are correct and some are not (since the observer could make a mistake, information transmission could introduce errors, etc.), while

- in case of some formulas it is not clear, whether they are true or false.

In summary, in the decision making process some pieces of knowledge are related to atomic formulas and the truth values of these formulas (and the status showing whether or not a truth value exists). The decision processes are often divided into 
different parts (for example, because some headquarters has divided decisions based on staff section role). It is therefore useful to "label" or "index" the above mentioned objects, in order to be able to "retrieve" them when needed.

\section{Knowledge in a Digital Circuit}

In this work we use the basic concepts and results of Lorents [5, 10, 11, 12, 13] to deal with knowledge. Therefore, we call knowledge every such ordered pair $\langle\mathrm{A}, \mathrm{B}\rangle$, where $\mathrm{A}$ is the notation (symbol, sign, etc.) for B and, at the same time, B is the denotation (meaning) of $\mathrm{A}$. In that case we say that $\mathrm{A}$ and $\mathrm{B}$ have a notationdenotation relationship and we represent this as A $\int \mathrm{B}$. The binary relation "J" has several natural algebraic properties, such as well-foundedness, a specific antisymmetry, and so called ordinary transitivity [5, 10-13]. The representation of knowledge in the digital circuits discussed below is based on the latter. For the digital circuit, knowledge is an ordered pair where the denotation is some situation description text in the database (some atomic formula or its negation) and the notation is the corresponding ID. Next, the ID is related to a specific block in the digital circuit schema (represented in Figure 2) by the block's address. Therefore, we have the following chain of notations and denotations: (block address $[I D),\left(I D \int_{\text {corresponding }}\right.$ text or atomic formula in the database), (text]the situation described by the text).

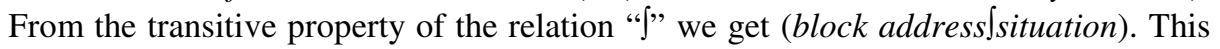
gets us finally the knowledge: 〈block address, situation〉. To some extent the above described can be related to the knowledge handling approaches from semiotics [see, for example, 14].

As mentioned above, one way to obtain correct knowledge is the application of the logical deduction apparatus (incl. correct and well-formed inference steps) [see 13, 15]. The technological realization of this has a role for both software and hardware, specifically the digital circuits described below.

\section{Software}

The implementation of the digital circuits below presumes the existence of suitable software. The software must enable inserting (situation description related) knowledge, the corresponding status and truth values. In addition, it must be possible to insert the axioms (provided by experts) that are required for the implementation of the deduction (inference) that supports the decision making process.

In order to achieve this we can use an SQL database and form three simple tables. The first table, A1, will contain knowledge in text form. Then a unique ID is automatically generated. This table must contain all the knowledge that will be operated with. Thereat, the ID and the corresponding knowledge (or the formula that represents it) are in a notation-denotation relationship (in other words, they form a new piece of knowledge on their own).

The second table, A2, will contain the expert-provided axioms. For example, the experts can claim that if there is a route from $\mathrm{X}$ to $\mathrm{Y}$ and at the same time there is a route from $\mathrm{Y}$ to $\mathrm{Z}$, then there must be a route from $\mathrm{X}$ to $\mathrm{Z}$, or shorter: $\mathrm{R}(\mathrm{X}, \mathrm{Y}) \& \mathrm{R}(\mathrm{Y}, \mathrm{Z}) \supset$ 
$\mathrm{R}(\mathrm{X}, \mathrm{Z})$. However, they may not tell us if there is or is not a route specifically between $\mathrm{X}$ and $\mathrm{Z}$. In this work we only use axioms that are represented by implications (or formulas in the form of $\mathrm{P} \supset \mathrm{Q}$ ). However, in this work we can only form implications based on knowledge that is present in table A1. In order to insert these axioms into table A2 we use the following quartet: 〈knowledge1 ID, knowledge2 ID, truth value 1 , truth value 2$\rangle$. For each inserted axiom a unique ID is generated.

The third table, A3, is filled (at the start of the decision process) by verifying that the information meets reality: is the inserted knowledge correct or not, or is it impossible to verify. If the knowledge is correct, then a 1 is inserted, if not correct, then a 0 is inserted. If verification was unsuccessful, then a NULL is inserted (basically, this "empty value" or NULL represents the third truth value discussed previously.

Since the digital circuits used in this work operate with only two distinct states $(0$ and 1), we must find a suitable solution for representing the NULL state in "electronic" form. In this work we have used the following solution:

A view A4 is automatically generated, which includes all of table A1 and the corresponding truth values from table A3. A column status is then added. If the TruthValue variable from table A3 was NULL, then a 0 is inserted in the column status. Otherwise (if the corresponding knowledge was found either true or false), the value of 1 is inserted. The column status cannot have any unfilled cells (all must have either 0 or 1). This way, we will get from table A4 a full overview of which pieces of knowledge are useable in the decision process, and which ones are not.

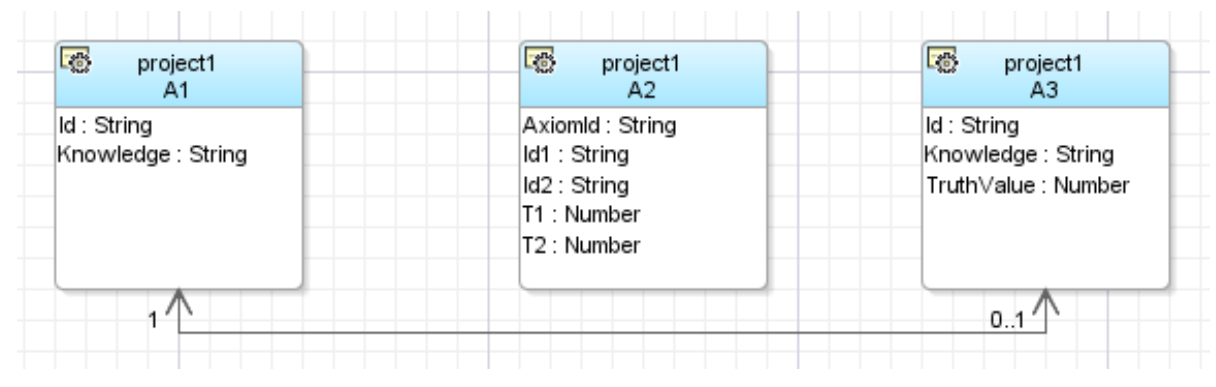

Fig. 1. Structure of tables

\section{Hardware}

Our long term goal is to create hardware, which

- loads all the data from the tables described above into its memory

- performs the knowledge verification using inference rules (finds the truth values for those knowledge related formulas that do not yet have truth values)

- provides the results to the user.

Next we describe the prototype of the simplified system. We start from the fact that the prototype can only transmit 8 bits at a time. In order to fulfill its "main purpose" (verify the correctness of the knowledge using inference rules), we must "inform" the 
circuit (using the information in the database) on whether it deals with knowledge from situation descriptions or from an expert-provided axiom; if the truth value of the knowledge is known or unclear; and if it is known, then what is it (true or false?), etc. We represent the corresponding information with sequence of the strings of bits of length eight:

$b_{18} b_{17} b_{16} b_{15} b_{14} b_{13} b_{12} b_{11}, b_{28} b_{27} b_{26} b_{25} b_{24} b_{23} b_{22} b_{21}, \ldots, b_{n 8} b_{n 7} b_{n 6} b_{n 5} b_{n 4} b_{n 3} b_{n 2} b_{n 1}, \ldots$

Let us clarify the role and meaning of those bits:

1. Knowledge input is represented by one string of bits (for example, $\left.b_{n 8} b_{n 7} b_{n 6} b_{n 5} b_{n 4} b_{n 3} b_{n 2} b_{n 1}\right)$.

2. Axiom input is represented by two consecutive strings of bits from our sequence (for example, $b_{n 8} b_{n 7} b_{n 6} b_{n 5} b_{n 4} b_{n 3} b_{n 2} b_{n 1}$ and $b_{n+18} b_{n+17} b_{n+16} b_{n+15} b_{n+14} b_{n+13} b_{n+12} b_{n+11}$ ). This is caused by the fact that according to our agreement axioms are only implications from knowledge. Each implication $(\mathrm{P} \supset \mathrm{Q})$ has two parts: the antecedent $(\mathrm{P})$ and consequent $(\mathrm{Q})$.

3. If $b_{n 1}=1$, then it is a case of knowledge input; if $b_{n 1}=0$, then it is a case of axiom input. The latter takes place as two consecutive inputs, where the first one is for the antecedent and the second one is for the consequent.

4. Next we look at the value of $b_{n 1}$. If $b_{n 1}=1$ (knowledge input), then $b_{n 2}$ represents the status of this knowledge: $b_{n 2}=1$ means that the knowledge truth value is known ( 0 or 1 ); $b_{n 2}=0$ means that the truth value is not known. However, if $b_{n 1}=0$ (axiom input), then $b_{n 2}=1$, since we assume that axioms provided by experts are correct (therefore it is also not possible to have an unclear truth value for an axiom).

5. Next we look at the values of $b_{n 1}$ ja $b_{n 2}$. If $b_{n 1}=1$ and $b_{n 2}=1$ (the correctness of the inserted knowledge is known, so we have the truth value $\mathrm{V}$ (where $\mathrm{V}=1$ or $\mathrm{V}=0$ ), then $b_{n 3}=V$. However, if $b_{n 1}=1$ ja $b_{n 2}=0$ (correctness of inserted knowledge is not known, so we do not have the truth value $\mathrm{V}$ ), then $b_{n 3}=0$. If $b_{n 1}=0$, then (as described previously) $b_{n 2}=1$, since it is a case of axiom input. Now (NB!) we must ascertain, whether the first or second component of the axiom is currently being inserted. If it is the first component, then we observe the antecedent $\mathrm{P}$ of the corresponding implication $\mathrm{P} \supset \mathrm{Q}$. If it is the second component, then the consequent $\mathrm{Q}$ is observed. The purpose of this observation is to determine if the formula starts with a negation. If it turns out that the formula $\mathrm{P}$ is the formula $\neg \mathrm{M}$, then $b_{n 3}=0$, otherwise ( $P$ is not the negation of some other formula) $b_{n 3}=1$. In the case of the second input the related string of bits $b_{n+18} b_{n+17} b_{n+16} b_{n+15} b_{n+14} b_{n+13} b_{n+12} b_{n+11}$ is observed as consequent Q. If formula $Q$ is the formula of $\neg N$, then $b_{n+13}=0$, otherwise ( $Q$ is not the negation of some other formula) $b_{n+13}=1$.

6. The next four bits $\left(b_{n 7} b_{n 6} b_{n 5} b_{n 4}\right)$ are meant for transmitting the address of the knowledge or axiom in question. For each piece of knowledge we use one address, and for each axiom we use two addresses (for example, 0001 for the antecedent and 0010 for the consequent of the first axiom). The address spaces for both axioms and pieces of knowledge start with 0001. The address increases by one for each new piece of knowledge and by two for each new axiom (using base two numbering).

7. The eighth bit is currently not used. 


\subsection{Input of Knowledge Related Info (Truth Value and Status)}

Depending on the value of the first bit $\left(b_{1}\right)$, the prototype must direct the input either to the knowledge or axiom elements.

First we describe knowledge input. The data is transferred from the database to the circuit bus by datapath (see Figure 2). The output Ln1 transmits information about whether the input data represents knowledge or an axiom. The out bus may provide additional information after the inference steps, which should be taken into account later (the prototype uses a multiplexer to choose between inputs from buses Bus $1 \_2$ and out). Before the inference process the out bus does not send any 1 signals (about status or truth value). If the address is not 0001 or if the data does not represent knowledge, then no input can be sent to the block in question (see Figure 2).

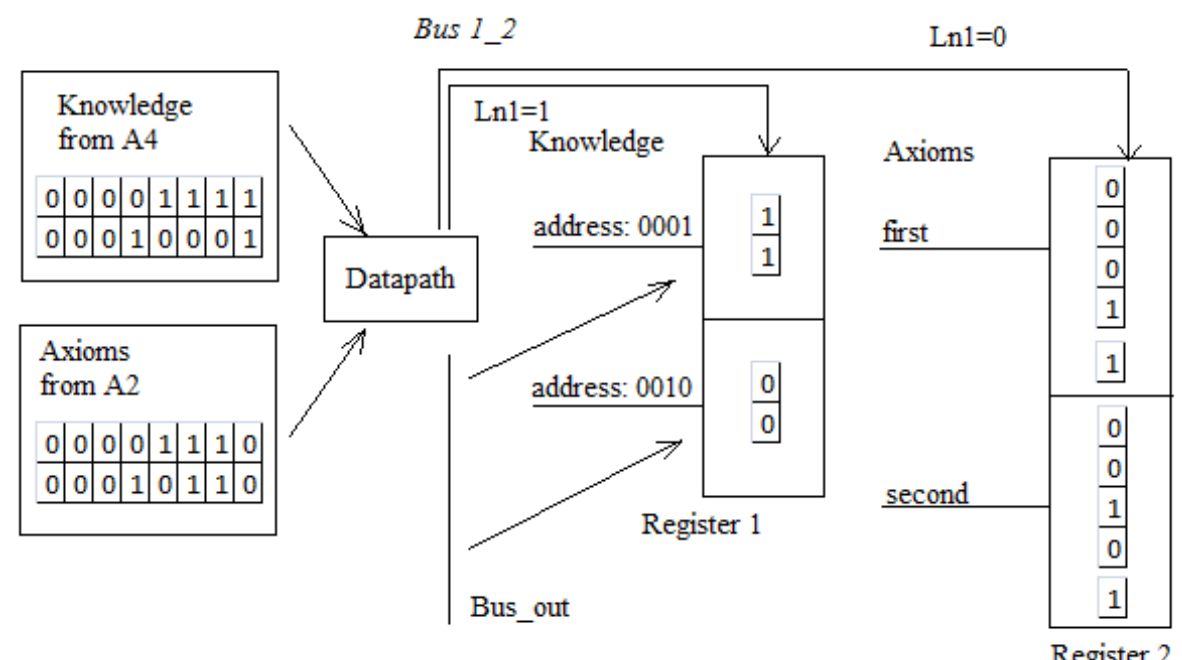

Fig. 2. Data input

\subsection{Input of Axiom Related Information}

If we wish to insert axiom (implication) related information to addresses 0001 and 0010, then the bus should deliver two bytes in our prototype: 00001110 and 00010110. The implication input block is equipped with a counter. In case of axioms it is important to keep in memory the address of the notation (address) of the antecedent or consequent.

\subsection{The Deduction Process in the Digital Circuit}

Next we briefly describe the most significant steps of the process that takes place in the circuit.

- The output of knowledge blocks (status and truth value) is sent to the buses. 
- If the status is 1 (we know the truth value), then suitable axioms are searched based on the truth value of the knowledge formulas. The axiom $\mathrm{A} \supset \mathrm{B}$ that fits to formula $F$ is searched as follows: the formula $F$ status (1) indicates that the truth value exists and it is either 0 or 1 . If the truth value of formula $F$ is 0 , then an axiom $A \supset B$ is considered fitting, if the consequent $\mathrm{B}$ is formula $\mathrm{F}$. If the truth value of formula $\mathrm{F}$ is 1 , then an axiom is $\mathrm{A} \supset \mathrm{B}$ is considered fitting, if the antecedent $\mathrm{A}$ is formula $\mathrm{F}$. Thereat, "just in case", the truth value (1) of the axiom is checked again (Fig.3).

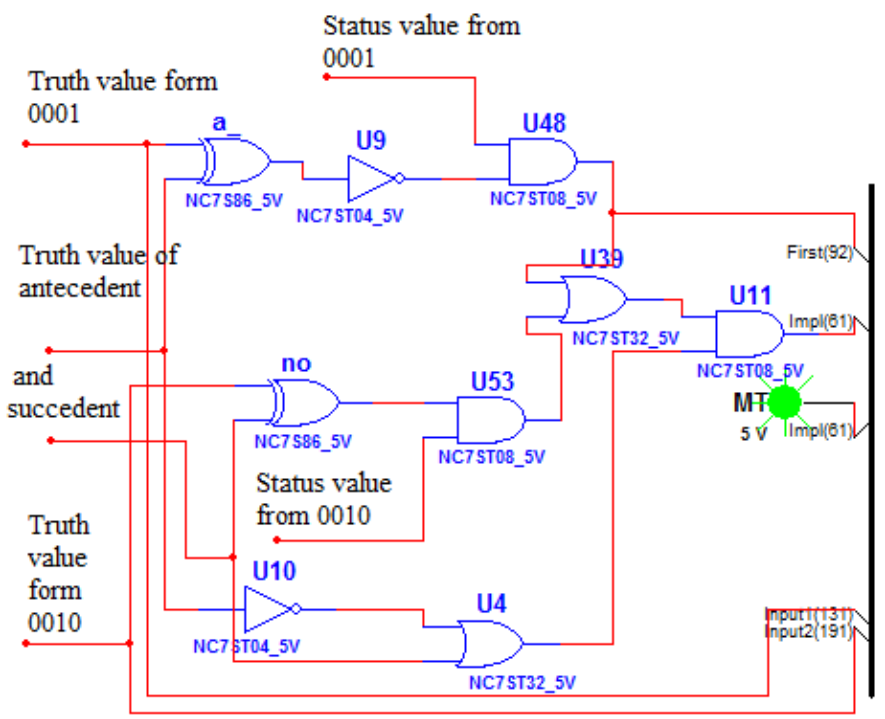

Fig. 3. Axiom search

- The set of inference rules implemented in the prototype is limited to Modus Ponens (MP) and Modus Tollens (MT). Therefore, a choice between the two must be made. If the formula $\mathrm{F}$ fits with the axiom $\mathrm{A} \supset \mathrm{F}$ (truth value of $\mathrm{F}$ equals 0 , and the truth value of formula $\neg \mathrm{F}$ equals 1 ), then MT is used. If the formula $\mathrm{F}$ fits with the axiom $\mathrm{F} \supset \mathrm{B}$ (truth value of formula $\mathrm{F}$ equals 1 ), then MP is used. The choice of inference rules is made with the help of 1-OF-10 DECODER. In order to activate the part of the circuit that implements the inference rule, a corresponding four bit input signal is generated. The first bit is 1 or 0 , depending on whether a fitting axiom was found for formula F. If the first bit is 1, then the next two bits represent the fitting axiom's antecedent's and consequent's status. If the first bit is 0 (no fitting axiom was found) then the next two bits are 0 . The fourth bit is always 0 .

- The part of the circuit that implements the inference rule MT generates a truth value of 0 for the antecedent of the axiom $\mathrm{A} \supset \mathrm{F}$ (which corresponds to the truth value of 1 for the formula $\neg \mathrm{A}$ ). The part of the circuit that implements MP generates the truth value of 1 for the consequent $B$ of $F \supset B$. Next a signal is generated, which carries information about either formula $\mathrm{A}$ (or $\neg \mathrm{A}$ ) or B: the status of the formula (1, due to the existence of the truth value) and the corresponding generated truth value. This information is sent to the database tables via the out bus (see Figure 1). 


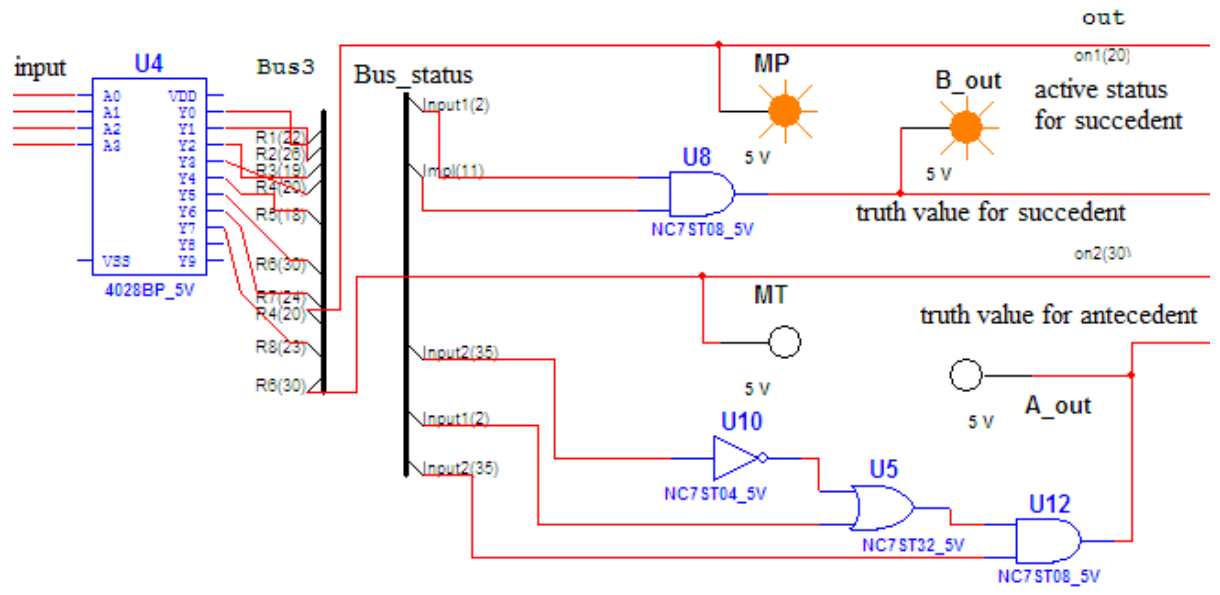

Fig. 4. Inference steps realization

\section{Summary}

The aim of this work was to study the options of using digital circuits for implementing inference steps that are necessary for the decision process. We described the prototype of one possible digital circuit solution and explained its operating concept. This prototype currently allows the use of only two inference rules (Modus Ponens and Modus Tollens). However, these are some of the most frequently used means for constructing the correct basis for correct decisions. The described prototype has been tested using the simulation software Multisim.

Acknowledgements. The authors would like to thank (NATO) CCD COE scientist Rain Ottis and Tallinn University of Technology Professor Peeter Ellervee for their assistance and support.

\section{References}

1. McCarthy, J., Hayes, P.J.: Some philosophical problems from the standpoint of Artificial Intelligence. In: Meltzer, B., Michie, D. (eds.) Machine Intelligence, vol. 4, pp. 463-502. Edinburgh University Press, Edinburgh (1969)

2. Kowalski, R., Sergot, M.: A logic-based calculus of events. New Generation Computing 4, 67-95 (1986)

3. Nguyen, T.D., Ho, T.B., Shimodaira, H.: A visualization tool for interactive learning of large decision trees. In: ICTAI 2000, pp. 28-35 (2000)

4. Renzi, M.F., Vicard, P., Guglielmetti, R., Musella, F.: Probabilistic expert systems for managing information to improve services. The TQM Journal 21(4), 429-442 (2009)

5. Lorents, P.: Knowledge and Logic. In: Proceedings of the International Conference on Artificial Intelligence, IC-AI 2009, July 13-16, 2009, vol. II, pp. 568-570. CSREA Press, Las Vegas (2009) 
6. Tarski, A.: Der Aussagenkalkül und die Topologie. Fund. Math. 31, 103-134 (1938)

7. Birkhoff, G., von Neumann, J.: The logic of quantum mechanics. Ann. Math. 37, 823-842 (1936)

8. Kleene, S.C.: Introduction to Metamathematics. Aimed at mathematicians. North Holland, Amsterdam (1952)

9. Goodstein, R.L.: Mathematical Logic. Leicester, England (1957)

10. Lorents, P.: Formalization of Data and Knowledge Based on the Fundamental Notationdenotation Relation. In: Proceedings of International Conference on Artificial Intelligence, vol. III, pp. 1297-1301. CSREA Press, Las Vegas (2001)

11. Lorents, P.: Denotations, Knowledge and Lies. In: Proceedings of the International Conference on Artificial Intelligence, IC-AI 2007, June 14-17, vol. II, pp. 324-329. CSREA Press, Las Vegas (2007)

12. Lorents, P.: Knowledge and Taxonomy of Intellect. In: Proceedings of the International Conference on Artificial Intelligence, IC-AI 2008, July 25-28, vol. II, pp. 484-489. CSREA Press, Las Vegas (2008)

13. Lorents, P.: Knowledge and Information. In: Proceedings of the 2010 International Conference on Artificial Intelligence, pp. 209-215. CSREA Press (2010)

14. Jastroch, N., Marlowe, T.: Knowledge Transfer in Collaborative Knowledge Management: A Semiotic View. In: 4th International Conference on Knowledge Generation, Communication and Management. KGCM, Orlando (2010)

15. Matsak, E., Lorents, P.: Digital solutions for inference rules in decision-supporting systems. In: Fifth International Conference on Digital Information Management. IEEE, Thundar Bay (2010) 